\title{
MicroRNA-199b promotes cell proliferation and invasion in Wilms' tumour by directly targeting Runt-related transcription factor 3
}

\author{
HUIZHEN ZHAO ${ }^{1 *}$, HAILING ZHAO $^{1 *}$, YONGNA ZHANG $^{1}$ and YUXI ZHOU ${ }^{2}$ \\ ${ }^{1}$ Department of Pediatrics, Yidu Central Hospital of Weifang, Weifang, Shandong 262550; \\ ${ }^{2}$ Department of Pediatrics, Weifang People's Hospital, Weifang, Shandong 261000, P.R. China
}

Received December 12, 2017; Accepted May 17, 2018

DOI: $10.3892 / \mathrm{mmr} .2018 .9096$

\begin{abstract}
Emerging evidence has demonstrated that the deregulation of microRNAs (miRNAs) contributes to Wilms' tumour (WT) malignant progression. Therefore, identifying the essential miRNAs for WT onset and progression may be a promising therapeutic method for patients with this disease. Dysregulation of miRNA-199b (miR-199b) serves significant roles in various types of human cancer. However, its expression patterns, possible functions and associated mechanisms in WT are largely unknown. In the present study, the expression of miR-199b in WT tissues was detected by reverse transcription-quantitative polymerase chain reaction (RT-qPCR) analysis. The biological functions of miR-199b overexpression in WT cells were determined using Cell counting kit- 8 and Transwell invasion assays. The mechanisms underlying the action of miR-199b in WT cells were also investigated using bioinformatics analysis, a luciferase reporter assay, RT-qPCR and western blot analysis. It was revealed that miR-199b expression was upregulated in WT tissues. In addition, the downregulation of miR-199b attenuated the proliferation and invasion of WT cells. Runt-related transcription factor 3 (RUNX3) was mechanistically predicted as a potential target of miR-199b. Subsequent experiments demonstrated that RUNX3 was a direct target gene of miR-199b in WT. In addition, the downregulation of RUNX3 in the WT tissues was inversely correlated with the miR-199b expression level. The recovered RUNX3 expression counteracted the oncogenic roles of miR-199b in WT cells. Therefore miR-199b may serve as an oncogene in WT progression by directly targeting
\end{abstract}

Correspondence to: Professor Yuxi Zhou, Department of Pediatrics, Weifang People's Hospital, 151 Guangwen Road, Weifang, Shandong 261000, P.R. China

E-mail: yuxi_zhou@yeah.net

*Contributed equally

Key words: Wilms' tumour, microRNA-199b, proliferation, invasion, runt-related transcription factor 3
RUNX3, thereby suggesting that the miR-199b/RUNX3 axis may be a promising therapeutic target for patients with WT.

\section{Introduction}

Wilms' tumour (WT), also known as renal embryonal tumour, is an embryonic cancer of the kidney that comprises blastemal, stromal and epithelial histological components (1). WT is the fourth most common renal tumour among paediatric cancers (2), and accounts for about $8 \%$ of all solid neoplasms and $95 \%$ of all renal carcinomas in children under 5 years old (3). Currently, nephrectomy, followed by chemotherapy and radiotherapy, are the major therapeutic methods for patients with WT (4). Significant improvements in diagnosis and treatment have improved the prognosis of patients with WT with localised disease. However, numerous patients suffer from metastasis or recurrence of the tumour; therefore, poor therapeutic outcomes are inevitable (5). Genetic and epigenetic changes, including the activation of oncogenes and/or the inhibition of tumour suppressors, are involved in tumorigenesis and tumour development of WT (6-8). Therefore, full investigation on the pathogenesis mechanisms of WT, particularly on the regulatory roles in the rapid growth and metastasis of WT cells, may be critical for development of novel therapeutic targets for treating patients with WT.

MicroRNAs (miRNAs) are clusters of noncoding, evolutionarily conserved and short RNA molecules implicated in gene expression (9). miRNAs can regulate gene expression by base pairing with complementary nucleotides in the 3'-untranslated regions of their target genes, thereby inducing mRNA degradation or translation suppression (10). Computational estimations indicate that over 1000 miRNAs existing in the human genome can regulate approximately $30 \%$ of the human protein-encoding genes (11). Furthermore, dysregulation of miRNAs is related with a substantial number of human disorders, including malignancies (12). Emerging evidence has highlighted that aberrantly expressed miRNAs may have significant effects on the occurrence and development of malignant tumours through acting either as oncogenes or tumour suppressors $(13,14)$. Upregulation of oncogenic miRNAs and/or suppression of tumour suppressive miRNAs can regulate various cancer-related biological features, such 
as cell proliferation, cycle, apoptosis, migration, invasion and metastasis (15). Hence, miRNAs may be utilised as effective biomarkers for cancer diagnosis, treatments and prognosis.

miR-199b is dysregulated in various types of human cancer, such as colorectal cancer (16), acute myeloid leukaemia (17), non-small cell lung cancer (18) and endometrioid endometrial carcinoma (19). However, miR-199b expression patterns, possible functions and associated mechanisms in WT remain predominantly unknown. Hence, miR-199b expression level in WT was detected in this study, and the regulatory role and underlying mechanisms of miR-199b on the biological features in WT were also investigated.

\section{Materials and methods}

Human tissue samples. This study was approved by the Ethics Committee of Yidu Central Hospital of Weifang. Written informed consent was also obtained from all patients. A total of 24 paired WT and adjacent normal tissues were collected from the patients diagnosed with WT and underwent nephrectomy at the Yidu Central Hospital of Weifang between January 2014 and November 2016. None of these patients with WT had been treated with chemotherapy or radiotherapy prior to nephrectomy. All the tissue specimens were immediately frozen and kept in liquid nitrogen until further RNA isolation.

Cell culture and oligonucleotide transfection. 17.94 and WiT49 cell lines were purchased from Shanghai ran Tai Biological Technology Co., Ltd. (Shanghai, China), and was cultured in Dulbecco's modified Eagle's medium (DMEM) containing $15 \%$ heat-inactivated fetal bovine serum (FBS) and $1 \%$ penicillin/streptomycin (all from Gibco, Carlsbad, CA, USA). WiT49 cell line was maintained in DMEM supplemented with $10 \%$ FBS and 1\% penicillin/streptomycin. 17.94 and WiT49 All cell lines were grown in a humidified incubator with $5 \% \mathrm{CO}_{2}$ and $95 \%$ air at $37^{\circ} \mathrm{C}$.

The miR-199b inhibitor and negative control (NC) miRNA inhibitor were acquired from GenePharma Co., Ltd (Shanghai, China). The specific small interfering RNA (siRNA) targeting the expression of Runt-related transcription factor 3 (RUNX3) and corresponding NC siRNA were provided by RiboBio Co., Ltd. (Guangzhou, China). Cells were plated in 6-well plates (1.5x105 cells/well) and transfected with miRNA inhibitor or siRNA using Lipofectamine ${ }^{\mathrm{TM}} 2000$ (Invitrogen; Thermo Fisher Scientific, Inc., Waltham, MA, USA), in accordance with the manufacturer's protocols. Afterwards, the transfected cells were maintained at $37^{\circ} \mathrm{C}$ in a cell incubator containing $5 \% \mathrm{CO}_{2}$, and the medium was changed to fresh Maccyo'5 supplemented with $10 \%$ FBS at 6 h post-transfection.

Reverse transcription-quantitative polymerase chain reaction (RT-qPCR) analysis. Total RNA of tissue samples or cells was isolated with TRIzol ${ }^{\circledR}$ reagent (Invitrogen; Thermo Fisher Scientific, Inc.) following the manufacturer's instructions. A NanoDrop 2000/2000c (Thermo Fisher Scientific, Inc., Wilmington, DE, USA) was used to detect the concentration of total RNA. For determination of miR-199b expression level, complementary DNA (cDNA) was synthesised from total RNA using a TaqMan MicroRNA Reverse Transcription kit. Subsequently, the qPCR was conducted with a TaqMan
MicroRNA PCR kit (all from Applied Biosystems, Carlsbad, CA, USA) and performed on Roche Lightcycler 480 Real-time PCR system (Roche Diagnostics, Basel, Switzerland). The cycling conditions for qPCR were as follows: $50^{\circ} \mathrm{C}$ for $2 \mathrm{~min}$, $95^{\circ} \mathrm{C}$ for $10 \mathrm{~min} ; 40$ cycles of denaturation at $95^{\circ} \mathrm{C}$ for $15 \mathrm{sec}$; and annealing/extension at $60^{\circ} \mathrm{C}$ for $60 \mathrm{sec}$. To analyse RUNX3 mRNA level, total RNA underwent reverse transcription into cDNA using a PrimeScript RT Reagent kit followed by qPCR with a SYBR Premix Ex Taq ${ }^{\mathrm{TM}}$ kit (all from Takara Bio, Dalian, China). The cycling conditions for qPCR were as follows: 5 min at $95^{\circ} \mathrm{C}$, followed by 40 cycles of $95^{\circ} \mathrm{C}$ for $30 \mathrm{sec}$ and $65^{\circ} \mathrm{C}$ for $45 \mathrm{sec}$. U6 snRNA and GAPDH were employed as internal control for miR-199b and RUNX3 mRNA, respectively. The primers were designed as follows: miR-199b forward, 5'-GCC CGCCCAGTGTTTAGACTAT-3' and reverse, 5'-GTGCAG GGTCCGAGGT-3'; U6 forward, 5'-GCTTCGGCAGCACAT ATACTAAAAT-3' and reverse, 5'-CGCTTCACGAATTTG CGTGTCAT-3'; RUNX3 forward, 5'-GACAGCCCCAACTTC CTCT-3' and reverse, 5'-CACAGTCACCACCGTACCAT-3'; and GAPDH forward, 5'-CGGAGTCAACGGATTTGGTCG TAT-3' and reverse, 5'-AGCCTTCTCCATGGTGGTGAA GAC-3'. Relative gene expression was calculated with the $2^{-\Delta \Delta C t}$ method (20).

Cell counting kit-8 (CCK-8) assay. Transfected cells were collected $24 \mathrm{~h}$ post-transfection and plated onto 96 well plates with a density of 3,000 cells for each well. Following incubation at $37^{\circ} \mathrm{C}$ for $0,24,48$ or $72 \mathrm{~h}, \mathrm{CCK}-8$ assay was carried out according to the manufacturer's instructions. After addition of $10 \mu$ l CCK-8 reagent (Dojindo Molecular Technologies, Inc., Kumamoto, Japan) into each well, the wells were incubated at $37^{\circ} \mathrm{C}$ with $5 \% \mathrm{CO}_{2}$ and $95 \%$ air for another $2 \mathrm{~h}$. Afterwards, absorbance was determined at a wavelength of $450 \mathrm{~nm}$ with an automatic multiwell spectrophotometer (Bio-Rad Laboratories, Inc., Hercules, CA, USA).

Transwell invasion assay. Cell invasion ability was detected using $8 \mu \mathrm{m}$ pore Boyden chambers (Corning Inc., Corning, NY, USA) coated with Matrigel (BD Biosciences, Franklin Lakes, NJ, USA). The transfected cells were collected, suspended in Maccyo'5 medium without FBS and counted under a light microscope (IX71; Olympus Co., Tokyo, Japan). A total of $5 \times 10^{4}$ cells were seeded into the upper chambers, whereas the Maccyo'5 medium containing 20\% FBS was added into the lower chambers. Subsequently, the cells were incubated at $37^{\circ} \mathrm{C}$ with $5 \% \mathrm{CO}_{2}$ for $24 \mathrm{~h}$. The noninvaded cells were removed with a cotton swab, whereas the invaded cells were fixed in methanol at room temperature for $15 \mathrm{~min}$ and stained with $0.5 \%$ crystal violet at room temperature for $15 \mathrm{~min}$. Finally, the stained cells were photographed and counted under an inverted light microscope (magnification, x200) with 5 randomly selected fields per chamber.

Bioinformatics prediction and luciferase reporter assay. TargetScan (www.targetscan.org) and microRNA.org (www.microrna.org/microrna/home.do) were utilised to predict the putative targets of miR-199b. Luciferase reporter plasmids, psiCHECK2-RUNX3-3'-UTR wild-type and psiCHECK2-RUNX3-3'-UTR mutant were synthesised and confirmed by GenePharma Co., Ltd. The 17.94 and 


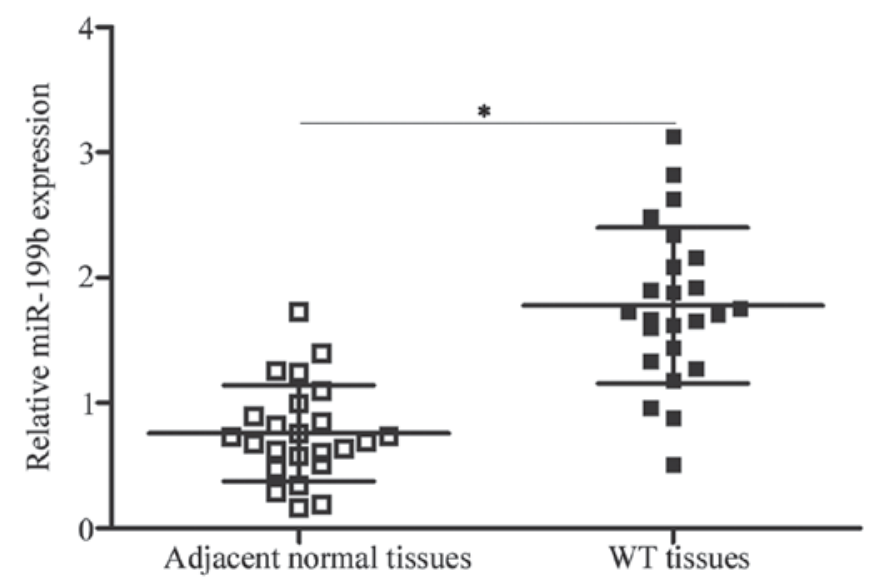

Figure 1. miR-199b expression is upregulated in WT tissues. The total RNA of 24 paired WT and adjacent normal tissues was isolated and reverse transcription-quantitative polymerase chain reaction was performed to detect miR-199b expression. " $\mathrm{P}<0.05$ vs. adjacent normal tissues. WT, Wilms' tumour; miR, microRNA.

WiT49 cells were transfected with miR-199b or NC inhibitor in combination with psiCHECK2-RUNX3-3'-UTR wild-type or psiCHECK2-RUNX3-3'-UTR mutant using Lipofectamine ${ }^{\mathrm{TM}} 2000$, according to the manufacturer's instructions. At $48 \mathrm{~h}$ post-transfection, luciferase activity in the cotransfected cells was measured using Dual-Luciferase Reporter Assay System (Promega, Madison, WI, USA), and renilla luciferase activity was normalised to the firefly luciferase activity.

Protein extraction and western blot analysis. Total protein was distilled from tissues or cells using the RIPA lysis buffer (Beyotime Institute of Biotechnology, Haimen, China). Afterwards, it was subjected to concentration determination using a BCA Protein Assay kit (Thermo Fisher Scientific, Inc., Waltham, MA, USA). Equal amounts of the total protein were resolved using 10\% SDS-PAGE and were transferred onto the polyvinylidene fluoride membranes (EMD Millipore, Billerica, MA, USA). After blocking with 5\% fat-free milk at room temperature for $1 \mathrm{~h}$, the membranes were incubated with primary antibodies at at $4{ }^{\circ} \mathrm{C}$ overnight, followed by further incubation with goat anti-rabbit horseradish peroxidase-conjugated secondary antibody (ab205718; 1:5,000 dilution; Abcam, Cambridge, MA, USA) at room temperature for $1 \mathrm{~h}$. After washing with Tris-buffered saline with Tween, the protein signals were visualised using an enhanced chemiluminescence kit (EMD Millipore). The primary antibodies used in this study included rabbit anti-human RUNX3 monoclonal antibody (ab49117; 1:1,000 dilution) and rabbit anti-human GAPDH monoclonal antibody (ab181602; 1:1,000 dilution; both Abcam). GAPDH was used as an internal control.

Statistical analysis. All data are expressed as the mean \pm standard deviation. SPSS (version 11.0; SPSS, Inc., Chicago, IL, USA) was used to carry out all the statistical analyses. Differences between groups were analysed with Student's t-test or one-way ANOVA, followed by Student-Newman-Keuls test. The association between miR-199b and RUNX3 mRNA in the WT tissues was evaluated using Spearman's correlation analysis. $\mathrm{P}<0.05$ was considered to indicate a statistically significant difference.

\section{Results}

miR-199b is upregulated in the WT tissues. The expression pattern of miR-199b in WT was determined by detecting its expression in 24 paired WT and adjacent normal tissues. Using RT-qPCR, miR-199b was significantly overexpressed in the WT tissues in comparison with that in the adjacent normal tissues (Fig. 1) $(\mathrm{P}<0.05)$. This result suggests that the dysregulation of miR-199b may be associated with WT progression.

Downregulation of $\mathrm{miR}-199 \mathrm{~b}$ inhibits cell proliferation and invasion in WT. To characterise the biological functions of miR-199b expression in WT, the 17.94 and WiT4917.94 and WiT49 cells were transfected with miR-199b inhibitor to knock down the endogenous miR-199b expression. Subsequent RT-qPCR analysis revealed that miR-199b was downregulated in the 17.94 and WiT49 cells that were transfected with miR-199b inhibitor in comparison with those cells transfected with $\mathrm{NC}$ inhibitor (Fig. 2A and B) $(\mathrm{P}<0.05)$. Moreover, CCK-8 assays were performed to explore the effect of miR-199b on WT cell proliferation. As shown in Fig. 2C and D, inhibition of miR-199b significantly reduced the 17.94 and WiT49 cell proliferation $(\mathrm{P}<0.05)$. Furthermore, the invasion capacity of the 17.94 and WiT49 cells following transfection with miR-199b or NC inhibitor was determined using Transwell invasion assay. The underexpression of miR-199b remarkably weakened the invasion ability of the 17.94 and WiT49 cells (Fig. 2E) $(\mathrm{P}<0.05)$. Therefore, miR-199b may act as an oncogene in WT progression.

RUNX3 is a direct target of miR-199b in WT. To understand the molecular mechanism underlying the oncogenic roles of miR-199b in WT, bioinformatics analysis was performed to predict the potential targets of miR-199b. RUNX3 (Fig. 3A), which is a well-known tumour suppressor, was predicted as a major target of miR-199b; thus, we selected it for further confirmation. To confirm this hypothesis, we transfected the 17.94 and WiT49 cells with miR-199b or NC inhibitor in combination with psiCHECK2-RUNX3-3'-UTR wild-type or psiCHECK2-RUNX3-3'-UTR mutant. The luciferase reporter assays showed that miR-199b downregulation significantly increased the luciferase activity of the psiCHECK2-RUNX3-3'-UTR wild-type in the 17.94 and WiT49 cells $(\mathrm{P}<0.05)$, whereas the luciferase activity was not significantly affected in the psiCHECK2-RUNX3-3'-UTR mutant (Fig. 3B and C). In addition, the RUNX3 expression was significantly increased at both mRNA (Fig. 3D) $(\mathrm{P}<0.05)$ and protein (Fig. 3E) $(\mathrm{P}<0.05)$ levels when miR-199b was knocked down in the 17.94 and WiT49 cells. Thus, RUNX3 is a direct target gene of miR-199b in WT.

RUNX3 is underexpressed in WT and is inversely correlated with the miR-199b level. The RUNX3 mRNA level in WT and adjacent normal tissues were determined to further illustrate the association between miR-199b and RUNX3 in WT. The expression level of RUNX3 mRNA was lowly expressed in the WT tissues compared with that in the adjacent normal 

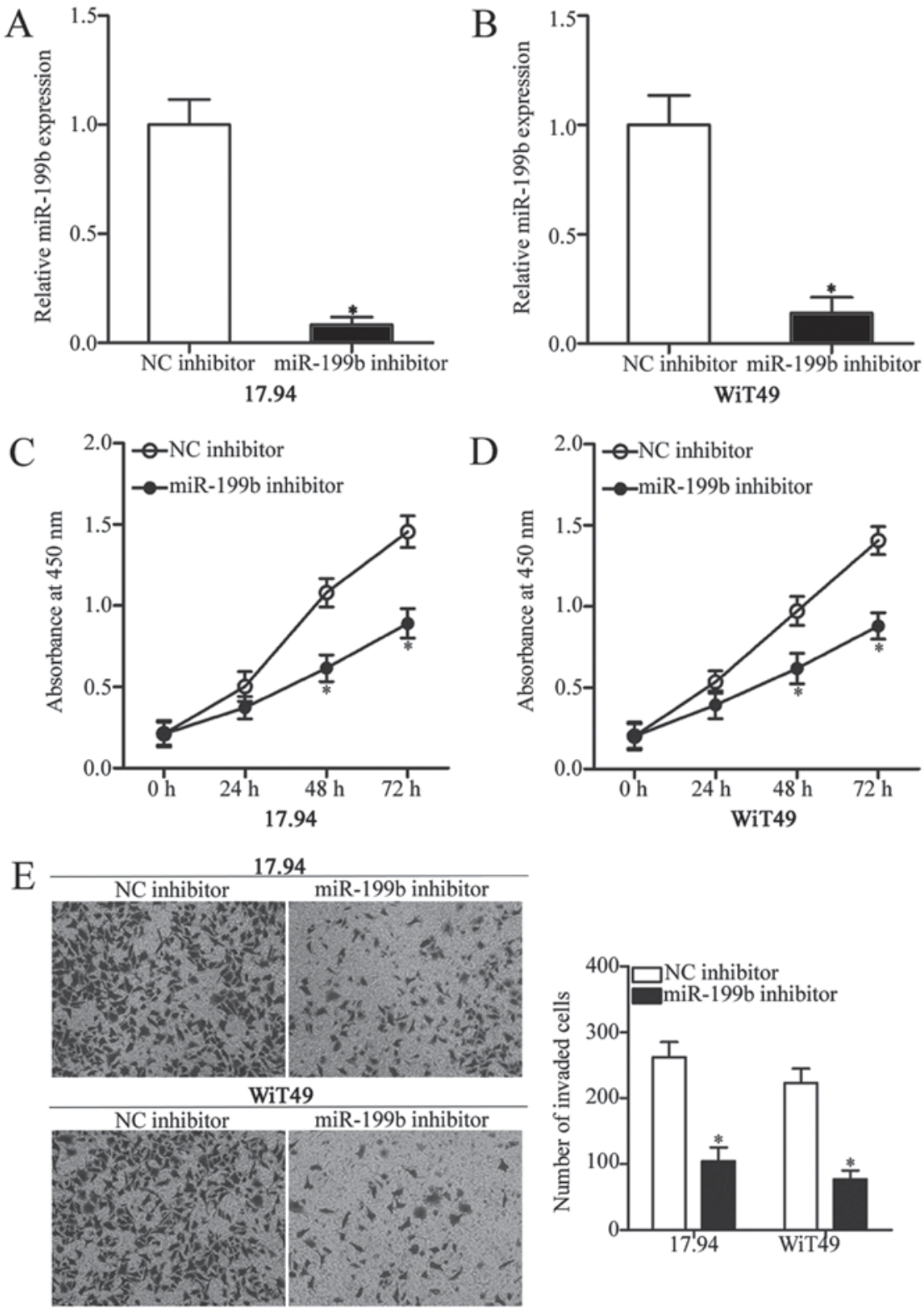

Figure 2. miR-199b knockdown suppresses the proliferation and invasion of 17.94 and WiT49 cells. miR-199b expression in (A) 17.94 and (B) WiT49 cells transfected with miR-199b or NC inhibitor were determined by reverse transcription-quantitative polymerase chain reaction. CCK- 8 assays were conducted to assess the proliferation of (C) 17.94 and (D) WiT49 cells following transfection with miR-199b or NC inhibitor. (E) The invasion ability of 17.94 and WiT49 cells transfected with miR-199b inhibitor or NC inhibitor was determined using Transwell invasion assays. Magnification, $\mathrm{x} 200$. "P<0.05 vs. NC inhibitor. NC, negative control; miR, microRNA; CCK, cell counting kit.

tissues (Fig. 4A, P<0.05). Western blot analysis also revealed that the RUNX3 protein was downregulated in the WT tissues (Fig. 4B). Furthermore, Spearman's correlation analysis identified a negative association between miR-199b and RUNX3 mRNA levels in WT tissues (Fig. 4C) $(\mathrm{r}=-0.6007, \mathrm{P}=0.0019)$. The downregulation of RUNX3 in the WT tissues may at least be partly due to the upregulation of miR-199b, thereby suggesting that RUNX3 was a direct target of miR-199b.

siRNA interference of RUNX3 reverses the oncogenic effects of miR-199b in WT cells. To further clarify whether the RUNX3 downregulation mediated the oncogenic roles of miR-199b in WT, we cotransfected the miR-199b inhibitor with RUNX3 siRNA or NC siRNA into the 17.94 and WiT49 cells. In comparison with cells cotransfected with the miR-199b inhibitor and NC siRNA, the RUNX3 protein expression level was recovered in the 17.94 and WiT49 cells after cotransfection with RUNX3 siRNA (Fig. 5A, P<0.05). Next, CCK-8 and Transwell invasion assays showed that the RUNX3 knockdown counteracted the oncogenic effects of miR-199b in the proliferation (Fig. 5B and C) $(\mathrm{P}<0.05)$ and invasion (Fig. 5D) $(\mathrm{P}<0.05)$ of 17.94 and WiT49 cells. On the basis of the results mentioned above, miR-199b may serve oncogenic roles in WT, at least in part, by direct regulation of RUNX3 expression.

\section{Discussion}

Emerging evidence has demonstrated that the deregulation of miRNAs contributes to WT malignant progression $(2,21,22)$. Therefore, identifying the essential miRNAs for WT onset and 

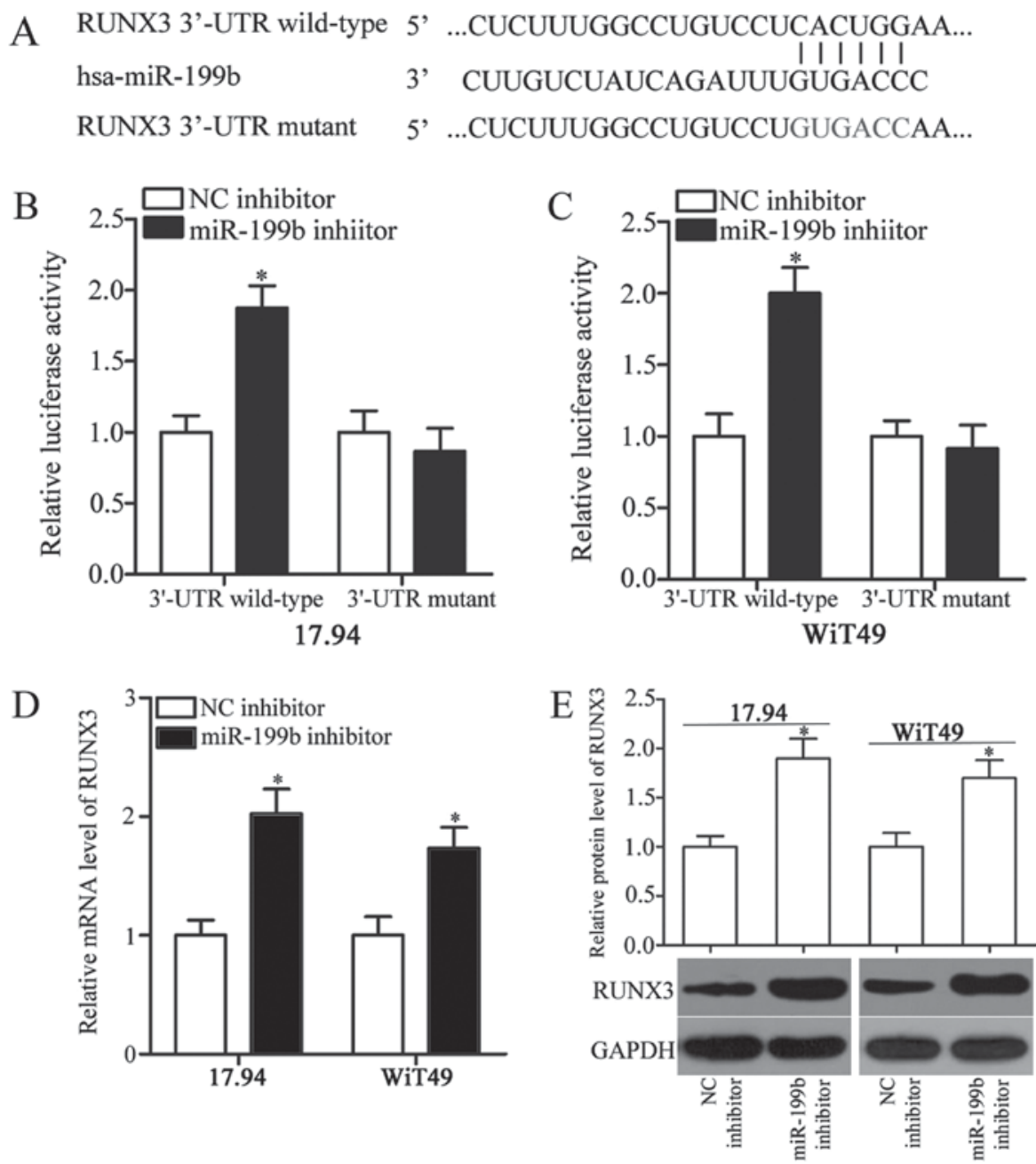

Figure 3. RUNX3 is a direct target of miR-199b in WT. (A) Wild-type and mutant binding sequences in the 3'-UTR of RUNX3 for miR-199b. (B) 17.94 and (C) WiT49 cells were cotransfected with miR-199b inhibitor or NC inhibitor and psiCHECK2-RUNX3-3'-UTR wild-type or psiCHECK2-RUNX3-3'-UTR mutant. After $48 \mathrm{~h}$ incubation the cells were harvested and the luciferase activity was analysed using a Dual-luciferase reporter assay system. (D) Reverse transcription-quantitative polymerase chain reaction and (E) western blot analysis were performed to detect the mRNA and protein expression of RUNX3, respectively in the 17.94 and WiT49 cells following transfection with miR-199b or NC inhibitor. ${ }^{*} \mathrm{P}<0.05$ vs. NC inhibitor. WT, Wilms' tumour; miR, microRNA; RUNX3, runt-related transcription factor 3; NC, negative control.
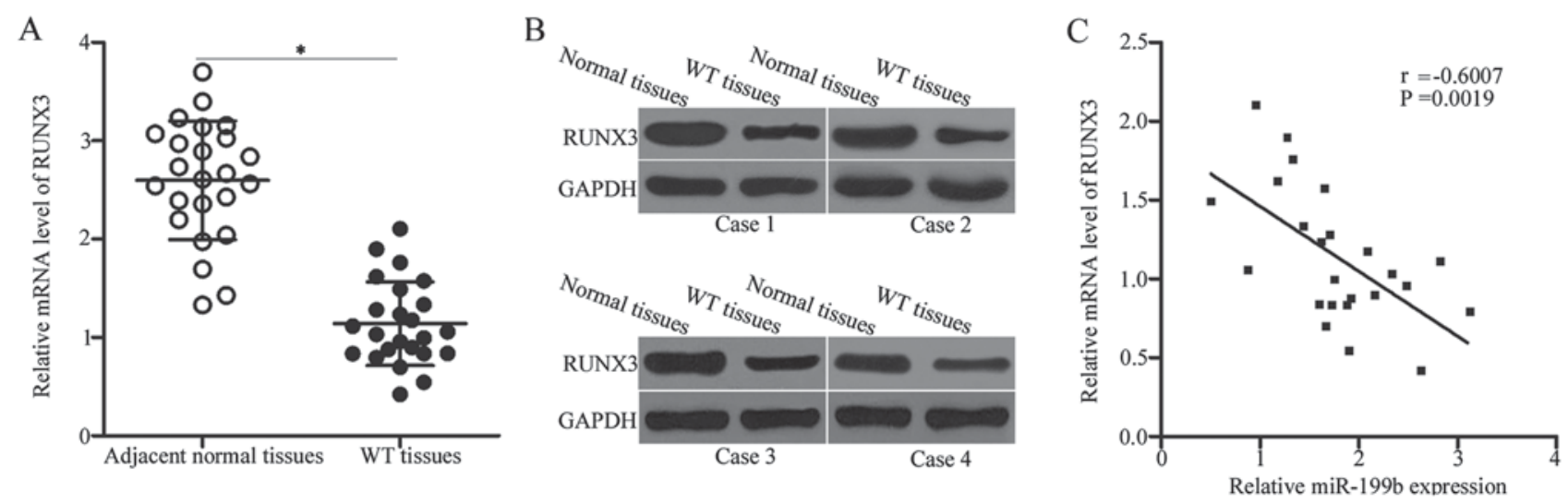

Figure 4. Downregulation of RUNX3 in WT tissues is negatively correlated with the miR-199b level. (A) Relative RUNX3 mRNA expression was lower in the WT tissues compared with the adjacent normal tissues. "P<0.05 vs. NC adjacent normal tissues. (B) Western blot analysis was performed to measure RUNX3 protein expression in WT and adjacent normal tissues. (C) Spearman's correlation analysis was applied to explore the association between the expression of miR-199b and RUNX3 mRNA in WT tissues. $\mathrm{r}=-0.6007, \mathrm{P}=0.0019$. WT, Wilms' tumour; miR, microRNA; RUNX3, runt-related transcription factor 3; NC, negative control.

progression may provide promising therapeutic methods for patients with this disease. In the current study, the expression of miR-199b in the WT tissues was significantly higher than that in the adjacent normal tissue. In addition, miR-199b 
A
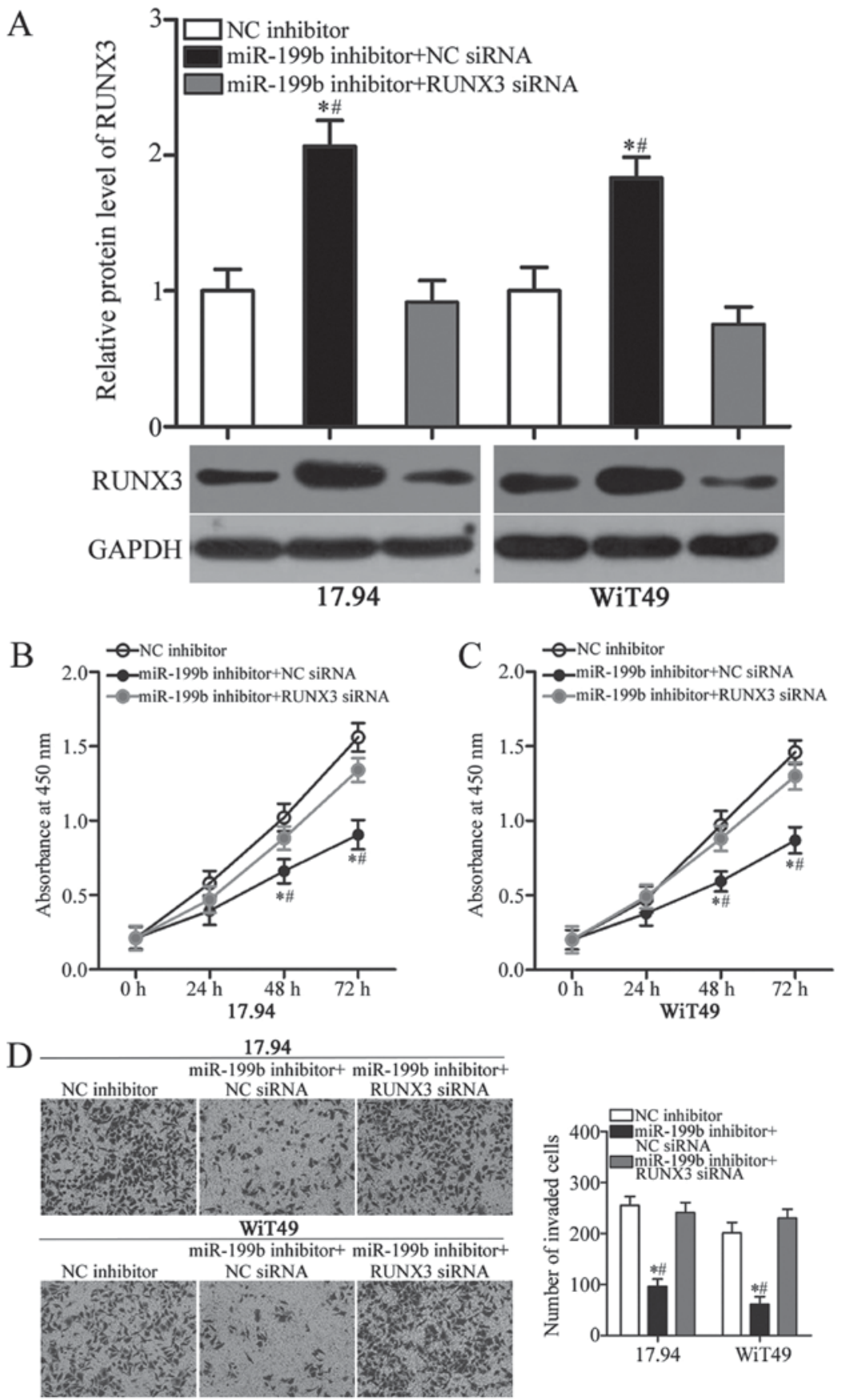

Figure 5. RUNX3 mediates the effects of miR-199b on WT cell proliferation and invasion. 17.94 and WiT49 cells were transfected with miR-199b inhibitor in combination with NC or RUNX3 siRNA. (A) At $72 \mathrm{~h}$ post-transfection, western blot analysis was performed to detect the RUNX3 protein expression. The proliferative ability of (B) 17.94 and (C) WiT49 cells and the (D) invasion ability of differently treated cells were assessed by CCK-8 and Transwell invasion assays, respectively. Magnification, $\mathrm{x} 200 .{ }^{~} \mathrm{P}<0.05$ vs. NC inhibitor. ${ }^{\prime} \mathrm{P}<0.05$ vs. miR-199b inhibitor+RUNX3 siRNA. WT, Wilms' tumour; miR, microRNA; RUNX3, runt-related transcription factor 3; NC, negative control; si, short interfering; CCK, cell counting kit.

underexpression reduced the WT cell proliferation and invasion. More importantly, RUNX3 was validated as a direct target gene of miR-199b in WT. Further studies showed that RUNX3 expression is lowly expressed in the WT tissues; and this expression is inversely associated with miR-199b level. Moreover, recovered RUNX3 expression counteracted the oncogenic effects of miR-199b underexpression in the WT cells. Thus, targeting miR-199b may be a novel therapeutic method for patients with WT.

In our study, we demonstrated that miR-199b expression is upregulated in WT. Similar phenomenon was also observed in osteosarcoma. Highly expressed miR-199b in osteosarcoma is evidently correlated with tumour grade, metastasis and recurrence. Osteosarcoma patients with increased miR-199b expression exhibit poorer overall survival and shorter disease-free survival relative to those patients with decreased levels (23). However, miR-199b is underexpressed in several human cancer types. For instance, the miR-199b expression level is decreased in hepatocellular carcinoma. Hepatocellular carcinoma patients with low miR-199b levels show shorter overall survival and progression-free survival rates than those patients with high miR-199b levels $(24,25)$. In bladder cancer, 
miR-199b expression is downregulated, which is associated with poor prognosis (26). In breast cancer, miR-199b expression is underexpressed in tumour tissues in comparison with normal breast tissues. Low miR-199b expression is strongly associated with TNM stage and lymph node metastasis of patients with breast cancer. Patients with breast cancer with relatively low miR-199b levels have poorer overall survival than those with high miR-199b level. Moreover, miR-199b is an independent prognostic factor for patients with breast cancer (27). miR-199b is also found to be lowly expressed in colorectal cancer (16), acute myeloid leukaemia (17), non-small cell lung cancer (18) and endometrioid endometrial carcinoma (19). These conflicting findings support the tissue specificity in the expression pattern of miR-199b and suggest that miR-199b may emerge as a potential novel biomarker for the diagnosis and prognosis in human cancers.

miR-199b serves oncogenic roles in WT by promoting cell proliferation and invasion. Similarly, miR-199b upregulation increases growth and metastasis of osteosarcoma cells in vitro (23). Nevertheless, miR-199b is found to play tumour suppressive roles in multiple human cancer types. For example, miR-199b overexpression suppresses cell proliferation and epithelial-mesenchymal transition, and increases radiosensitivity in hepatocellular carcinoma $(24,25)$. Fang et al found that miR-199b overexpression restrains cell growth, colony formation and metastasis $(27,28)$. Shen et al revealed that restored miR-199b expression represses the colorectal cancer cell metastasis in vitro and in vivo; and elevates the cell chemosensitivity to 5-FU and oxaliplatin (16). Wang et al found that the miR-199b resumption expression reduces the cell proliferative, migratory and invasive abilities of non-small cell lung cancer (18). Shang et al demonstrated that miR-199b re-expression attenuates cell proliferation and induces apoptosis in prostate cancer (29). Koshizuka et al indicated that enforced expression of miR-199b inhibits cell migration and invasion of the head and neck cancer (30). Thus, biological functions of miR-199b in human malignancy have tissue specificity, thereby suggesting that miR-199b may be an effective therapeutic target for patients with these types of human malignancy.

Several targets of miR-199b have previously been identified, including Hif $1 \alpha$ (24) and N-cadherin (25) in hepatocellular carcinoma, HER2 (28) in breast cancer, SIRT1 (16) in colorectal cancer, ZEB1 (18) in non-small cell lung cancer and ITGA3 (30) in head and neck cancer. In this study, RUNX3 was proven to be a direct target of miR-199b in WT. RUNX3 is downregulated in a variety of human cancers, including lung cancer (31), bladder cancer (32), breast cancer (33), gastric cancer (34) and colorectal cancer (35). The downregulation of RUNX3 accounts for the tumour initiation and progression by acting as a tumour suppressor and regulating numerous cancer-related biological processes, such as cell proliferation, cell cycle, apoptosis, metastasis and angiogenesis (36-39). Furthermore, the deregulation of RUNX3 is associated with malignant clinical features, thereby influencing the prognosis of patients with malignant diseases (40). For example, decreased RUNX3 in breast cancer is significantly correlated with tumour infiltration, clinical stage, lymph node metastasis and expressions of oestrogen and progesterone receptors. In addition, patients with breast cancer with higher RUNX3 expression have higher survival rate than patients with lower RUNX3 (33). These findings suggest that the ectopic expression of RUNX3 in human cancers may be a promising therapeutic method.

In conclusion, miR-199b expression is upregulated in WT. miR-199b inhibition restrains cell proliferation and invasion in WT by directly targeting RUNX3. These results suggest its potential as a therapeutic target to treat patients with WT. However, in our current study, we did not analyse the effects of miR-199b overexpression in WT cells. This is a limitation of our study, and we will resolve this in our future experiments. Moreover, we will explore whether miR-199b could directly targeted other genes in WT.

\section{Acknowledgements}

Not applicable.

\section{Funding}

No funding was received.

\section{Availability of data and materials}

The datasets used and/or analyzed during the present study are available from the corresponding author on reasonable request.

\section{Authors' contributions}

YZho and HuZ designed the study. HuZ and HaZ performed the RT-qPCR and western blot analysis, as well as the CCK-8 and Transwell invasion assays. YZha performed the luciferase reporter assay. YZho analyzed the data.

\section{Ethics approval and consent to participate}

The present study was approved by the Ethics Committee of Yidu Central Hospital of Weifang. Written informed consent was obtained from all patients prior to their inclusion within the study.

\section{Consent for publication}

Not applicable.

\section{Competing interests}

The authors declare that they have no competing interests.

\section{References}

1. Yan-Fang T, Zhi-Heng L, Li-Xiao X, Fang F, Jun L, Gang L, Lan C, Na-Na W, Xiao-Juan D, Li-Chao S, et al: Molecular mechanism of the cell death induced by the histone deacetylase pan inhibitor LBH589 (Panobinostat) in Wilms tumor cells. PLoS One 10: e0126566, 2015.

2. Cui M, Liu W, Zhang L, Guo F, Liu Y, Chen F, Liu T, Ma R and Wu R: Over-expression of miR-21 and lower PTEN levels in Wilms' tumor with aggressive behavior. Tohoku J Exp Med 242: 43-52, 2017. 
3. Beckwith JB, Kiviat NB and Bonadio JF: Nephrogenic rests, nephroblastomatosis, and the pathogenesis of Wilms' tumor Pediatr Pathol 10: 1-36, 1990.

4. Schenk JP, Günther P, Schrader C, Ley S, Furtwängler R Leuschner I, Edelhäuser M, Graf N and Tröger J: Childhood kidney tumors - the relevance of imaging. Radiologe 45: 1112-1123, 2005 (In German).

5. Routh JC, Grundy PE, Anderson JR, Retik AB and Kurek KC: B7-h1 as a biomarker for therapy failure in patients with favorable histology Wilms tumor. J Urol 189: 1487-1492, 2013.

6. Wang J, Li Y, Ma F, Zhou H, Ding R, Lu B, Zou L, Li J and Lu R Inhibitory effect of Par-4 combined with cisplatin on human Wilms' tumor cells. Tumour Biol 39: 1010428317716689, 2017.

7. Wari MN, Vallonthaiel AG, Ahmed A, Saxena D, Iyer VK, Mathur SR, Agarwala S, Bakhshi S, Srinivas V, Chattopadhyaya $\mathrm{P}$, et al: Glypican-3 mRNA expression level in Wilms tumor: Correlation with histological type, stage, and outcome. Pediatr Surg Int 33: 695-703, 2017.

8. Zhu S, Liu G, Fu W, Hu J, Fu K and Jia W: Axl promotes the proliferation, invasion and migration of Wilms' tumor and can be used as a prognostic factor. Onco Targets Ther 10: 955-963, 2017.

9. Lin S and Gregory RI: MicroRNA biogenesis pathways in cancer. Nat Rev Cancer 15: 321-333, 2015.

10. Baranwal S and Alahari SK: miRNA control of tumor cell invasion and metastasis. Int J Cancer 126: 1283-1290, 2010.

11. Liu J: Control of protein synthesis and mRNA degradation by microRNAs. Curr Opin Cell Biol 20: 214-221, 2008

12. Esquela-Kerscher A and Slack FJ: Oncomirs-microRNAs with a role in cancer. Nat Rev Cancer 6: 259-269, 2006.

13. Harada K, Baba Y, Ishimoto T, Shigaki H, Kosumi K, Yoshida N Watanabe $\mathrm{M}$ and Baba $\mathrm{H}$ : The role of microRNA in esophageal squamous cell carcinoma. J Gastroenterol 51: 520-530, 2016.

14. Bartel DP: MicroRNAs: Genomics, biogenesis, mechanism, and function. Cell 116: 281-297, 2004.

15. Calin GA and Croce CM: MicroRNA signatures in human cancers. Nat Rev Cancer 6: 857-866, 2006.

16. Shen ZL, Wang B, Jiang KW, Ye CX, Cheng C, Yan YC, Zhang JZ, Yang Y, Gao ZD, Ye YJ and Wang S: Downregulation of miR-199b is associated with distant metastasis in colorectal cancer via activation of SIRT1 and inhibition of CREB/KISS1 signaling. Oncotarget 7: 35092-35105, 2016.

17. Favreau AJ, McGlauflin RE, Duarte CW and Sathyanarayana $P$ miR-199b, a novel tumor suppressor miRNA in acute myeloid leukemia with prognostic implications. Exp Hematol Oncol 5: 4, 2016

18. Wang J, Zhou F, Yin L, Zhao L, Zhang Y and Wang J: MicroRNA-199b targets the regulation of ZEB1 expression to inhibit cell proliferation, migration and invasion in non-small cell lung cancer. Mol Med Rep 16: 5007-5014, 2017.

19. Torres A, Torres K, Pesci A, Ceccaroni M, Paszkowski T, Cassandrini P, Zamboni G and Maciejewski R: Deregulation of miR-100, miR-99a and miR-199b in tissues and plasma coexists with increased expression of mTOR kinase in endometrioid endometrial carcinoma. BMC Cancer 12: 369, 2012.

20. Livak KJ and Schmittgen TD: Analysis of relative gene expression data using real-time quantitative PCR and the 2(-Delta Delta C(T)) method. Methods 25: 402-408, 2001

21. Wang HF, Zhang YY, Zhuang HW and Xu M: MicroRNA-613 attenuates the proliferation, migration and invasion of Wilms tumor via targeting FRS2. Eur Rev Med Pharmacol Sci 21: 3360-3369, 2017

22. Liu GL, Yang HJ, Liu B and Liu T: Effects of MicroRNA-19b on the proliferation, apoptosis, and migration of Wilms' tumor cells via the PTEN/PI3K/AKT signaling pathway. J Cell Biochem 118 : 3424-3434, 2017.

23. Zeng H, Zhang Z, Dai X, Chen Y, Ye J and Jin Z: Increased expression of microRNA-199b-5p associates with poor prognosis through promoting cell proliferation, invasion and migration abilities of human osteosarcoma. Pathol Oncol Res 22: 253-260, 2016.
24. Wang C, Song B, Song W, Liu J, Sun A, Wu D, Yu H, Lian J, Chen L and Han J: Underexpressed microRNA-199b-5p targets hypoxia-inducible factor- $1 \alpha$ in hepatocellular carcinoma and predicts prognosis of hepatocellular carcinoma patients. J Gastroenterol Hepatol 26: 1630-1637, 2011

25. Zhou SJ, Liu FY, Zhang AH, Liang HF, Wang Y, Ma R, Jiang YH and Sun NF: MicroRNA-199b-5p attenuates TGF- $\beta 1$-induced epithelial-mesenchymal transition in hepatocellular carcinoma. Br J Cancer 117: 233-244, 2017.

26. Sakaguchi T, Yoshino H, Yonemori M, Miyamoto K, Sugita S, Matsushita R, Itesako T, Tatarano S, Nakagawa M and Enokida H: Regulation of ITGA3 by the dual-stranded microRNA-199 family as a potential prognostic marker in bladder cancer. Br J Cancer 116: 1077-1087, 2017.

27. Fang C, Wang FB, Li Y and Zeng XT: Down-regulation of miR-199b-5p is correlated with poor prognosis for breast cancer patients. Biomed Pharmacother 84: 1189-1193, 2016.

28. Fang C, Zhao Y and Guo B: MiR-199b-5p targets HER2 in breast cancer cells. J Cell Biochem 114: 1457-1463, 2013.

29. Shang W, Chen X, Nie L, Xu M, Chen N, Zeng H and Zhou Q: MiR199b suppresses expression of hypoxia-inducible factor $1 \alpha$ $(\mathrm{HIF}-1 \alpha)$ in prostate cancer cells. Int J Mol Sci 14: 8422-8436, 2013.

30. Koshizuka K, Hanazawa T, Kikkawa N, Arai T, Okato A, Kurozumi A, Kato M, Katada K, Okamoto Y and Seki N: Regulation of ITGA3 by the anti-tumor miR-199 family inhibits cancer cell migration and invasion in head and neck cancer. Cancer Sci 108: 1681-1692, 2017.

31. Araki K, Osaki M, Nagahama Y, Hiramatsu T, Nakamura H, Ohgi $\mathrm{S}$ and Ito $\mathrm{H}$ : Expression of RUNX3 protein in human lung adenocarcinoma: Implications for tumor progression and prognosis. Cancer Sci 96: 227-231, 2005.

32. Dodurga Y, Avci CB, Satiroglu-Tufan NL, Tataroglu C, Kesen Z, Doğan ZO, Yilmaz $S$ and Gündüz C: Detection of deleted in malignant brain tumors 1 and runt-related transcription factor 3 gene expressions in bladder carcinoma. Mol Biol Rep 39: 4691-4695, 2012

33. Jiang Y, Tong D, Lou G, Zhang Y and Geng J: Expression of RUNX3 gene, methylation status and clinicopathological significance in breast cancer and breast cancer cell lines. Pathobiology 75: 244-251, 2008.

34. Hsu PI, Hsieh HL, Lee J, Lin LF, Chen HC, Lu PJ and Hsiao M: Loss of RUNX3 expression correlates with differentiation, nodal metastasis, and poor prognosis of gastric cancer. Ann Surg Oncol 16: 1686-1694, 2009.

35. Zheng W, Zheng K, Zhong L, Li Q and Huang Z: Expression of Runx3 and C-myc in human colorectal cancer. Nan Fang Yi Ke Da Xue Xue Bao 34: 1042-1047, 2014 (In Chinese).

36. Jili S, Eryong L, Lijuan L and Chao Z: RUNX3 inhibits laryngeal squamous cell carcinoma malignancy under the regulation of miR-148a-3p/DNMT1 axis. Cell Biochem Funct 34: 597-605, 2016.

37. Chen F, Liu X, Cheng Q, Zhu S, Bai J and Zheng J: RUNX3 regulates renal cell carcinoma metastasis via targeting miR-6780a-5p/E-cadherin/EMT signaling axis. Oncotarget 8: 101042-101056, 2017.

38. Kim BR, Kang MH, Kim JL, Na YJ, Park SH, Lee SI, Kang S, Joung SY, Lee SY, Lee DH, et al: RUNX3 inhibits the metastasis and angiogenesis of colorectal cancer. Oncol Rep 36: 2601-2608, 2016.

39. Li H, Li D and Meng N: Effects of RUNX3 mediated Notch signaling pathway on biological characteristics of colorectal cancer cells. Int J Oncol 50: 2059-2068, 2017.

40. WeiD, Gong W, Oh SC,Li Q,Kim WD, Wang L,LeX, Yao J, Wu TT, Huang S and Xie K: Loss of RUNX3 expression significantly affects the clinical outcome of gastric cancer patients and its restoration causes drastic suppression of tumor growth and metastasis. Cancer Res 65: 4809-4816, 2005. 